\title{
Exploring Dark Energy with SNAP
}

\author{
G. Aldering ${ }^{1}$ \\ Physics Division, Lawrence Berkeley National Lab, Berkeley, CA
}

\begin{abstract}
The accelerating expansion of the Universe is one of the most surprising and potentially profound discoveries of modern cosmology. Measuring the acceleration well enough to meaningfully constrain interesting physical models requires improvements an order of magnitude beyond on-going and near-term experiments. The Supernova/Acceleration Probe has been conceived as a powerful yet simple experiment to use Type Ia supernovae and weak gravitational lensing to reach this level of accuracy. As fundamentally different causes for the acceleration map into very small differences in observational parameters for all relevant cosmological methods, control of systematics is especially important and so has been built into the SNAP mission design from the very beginning.

Though focused on the study of the accelerating Universe, the overall SNAP instrument suite is quite general and able to make unique contributions to a wide variety of astronomical studies. The baseline satellite consists of a $2-\mathrm{m}$ anastigmat telescope, with a 0.7 square degree focal plane paved with optical and NIR imaging arrays. Spectroscopy can be obtained using a high-throughput low-resolution optical+NIR integral field spectrograph. The baseline science programs will result in a 15 square degree "deep field" having temporal coverage every 4 days and summing to $m_{A B} \sim 30.3$ in all colors - to be used for discovery and follow-up of some 2000 Type Ia supernova in the range $0.1<z<1.7$ - and a wide area survey spanning 1000 square degrees and reaching $m_{A B} \sim 27.7$ in all colors - to be used to measure the weak lensing power spectrum well into the non-linear regime. A panoramic survey covering 10000 square degrees to $m_{A B} \sim 26.7$ in all colors is also possible. This baseline dataset represents a gold mine for archival astronomical research and follow-up with JWST, while guest observer survey programs will substantially broaden the impact that $S N A P$ will have.
\end{abstract}

1 The work described here is supported by the DOE Office of Science and by NASA. The author thanks his collaborators in SNAP and the SCP for their valuable contributions to this work.

Preprint submitted to Elsevier Science $\quad 27$ July 2021 


\section{Introduction}

$S N A P^{2}$ is a space telescope mission concept aimed at measuring the time variation in the equation of state of the dark energy responsible for the accelerating expansion of the universe. SNAP initially focused on the use of Type Ia supernovae as a means of measuring the expansion history of the universe through the luminosity-distance relation. However, the SNAP instrument suite proved to be quite generic, allowing, for example, the inclusion of gravitational weak lensing as another means of constraining the properties of dark energy. Indeed, $S N A P$ 's very powerful wide-field imager and the interest in using it for complementary science has in many ways motivated and shaped this "Wide-Field Imaging from Space" conference.

\section{Cosmology with Supernova from the Ground and Space}

Before looking at the exciting discoveries that $S N A P$ holds for the future, it is worthwhile to consider recent past and on-going work to constrain the nature of dark energy. In the six years since the discovery of the accelerating expansion of the universe a wealth of new observations have confirmed and further refined the initial discovery. In concert with newer data from Type Ia supernovae (including constraints on flatness from the CMB and on $\Omega_{M}$ from large-scale structure and clusters), the statistical significance of the acceleration is now $>10 \sigma$. Just as importantly, the equation of state of the dark energy is now constrained to be $w<-0.7$ (assuming the equation of state is constant).

Space-based observations have played an increasing role in cosmological measurements of Type Ia supernovae. This is simply due to the much smaller sky noise present in a point-source photometry aperture in space compared with the ground - especially at redder optical wavelengths. The discovery papers $[11,15]$ contained just four supernovae measured with HST. Samples totaling 39 Type Ia supernovae, segregated into early, middle and late host-galaxy morphologies from an HST Snapshot survey, were shown to support an accelerating expansion [20]. Precise corrections for dust extinction of eleven more supernovae made possible by accurate HST photometry were shown to support the accelerating expansion and provided tighter constraints on the dark energy equation of state [5]. A sample of high-redshift supernovae reaching $z \sim 1.6$ has shown that the supernovae follow the transition from deceleration to acceleration expected as the universe goes from matter domination to dark energy domination $[16,5,18]$. These space-based measurements have significantly bolstered both the case for dark energy and the efficacy of Type Ia

2 snap.lbl.gov; see also [1] 

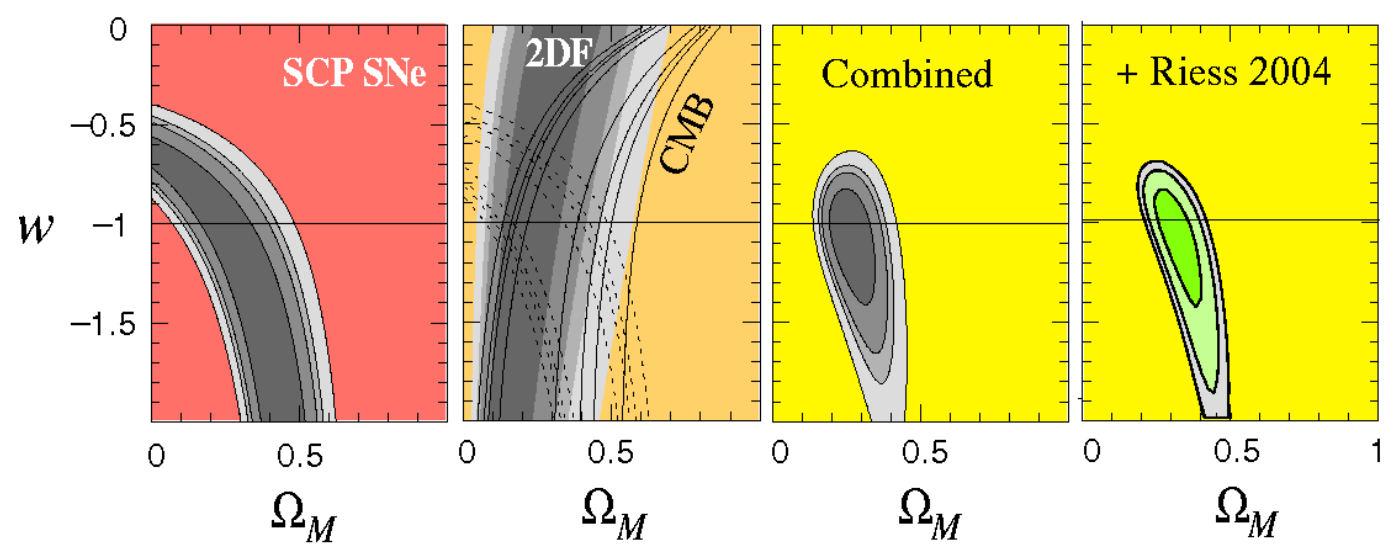

Fig. 1. The most recent constraints on the dark energy equation of state, $w$. A constant equation of state and flat topology are assumed. Inner and outer contours delimit $68 \%$ and $99 \%$ confidence, respectively. On the left, the constraints from the Supernova Cosmology Project sample [9-11,5] are shown. These include eleven supernovae with very well-measured colors from $H S T$. The next panel shows independent constraints from WMAP [17] and from the 2dF redshift survey [8]. The third panel shows the results of combining these independent measurements. The right-most panel shows the effect of the improved constraints on $\Omega_{M}$ from the Type Ia supernovae in the deceleration epoch resulting from the addition of supernovae from the Higher- $Z$ Supernova Search Team [16].

supernovae as distance indicators. Accordingly, today the most precise and highest-redshift supernova measurements — several dozen so far — are most often obtained with $H S T$.

An example of the state of the art in space-based supernova cosmology is a joint endeavor involving the Supernova Cosmology Project $\left(\mathrm{SCP}^{3}\right.$ ) and the Higher- $Z$ Supernova Search Team (HZSST) which around the time of this conference was conducting a search spanning four epochs, each covering fifteen pointings covering the northern GOODS field. The searches are separated by 45 days - roughly equal to the time from explosion to maximum light in the restframe of $z>1$ Type Ia supernovae. The search uses the Advanced Camera for Surveys (ACS) with the $850 \mathrm{~nm}$ long-pass filter in order to reach redward of the restframe UV cut-off exhibited by Type Ia supernovae. A short exposure in the F775W filter is added in order to reject some fraction of Type II supernovae, which in the first 10 days have much more UV flux than Type Ia supernovae. The depth of the GOODS field at optical (HST) and infrared (ground-based) wavelengths means that photometric redshifts are known for many supernova host galaxies. In addition, the galaxy colors at the location of the supernovae provide a handle on whether or not star-formation is likely to be ongoing, which can help eliminate likely core-collapse supernovae. All of this

3 supernova.lbl.gov 


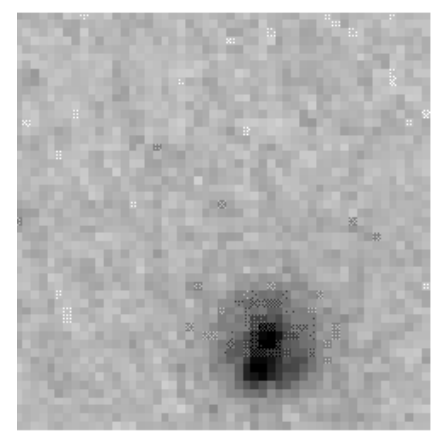

Reference

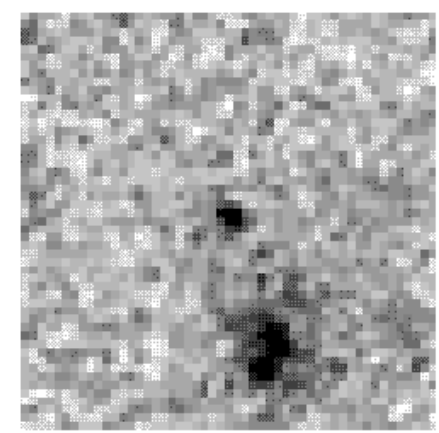

New

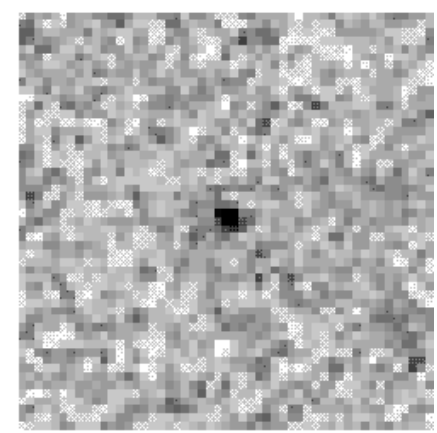

Discovery

Fig. 2. Discovery image of a candidate $z \sim 1.7$ Type Ia supernova from a search of the HDF-N with HST by the SCP. The image on the left is the reference image constructed from the GOODS program. The central panel shows the discovery image, obtained in one $H S T$ orbit in the F850LP filter. The image on the right is the difference of the discovery and reference images, isolating the new supernova. The redshift is based on photometric redshifts kindly provided by GOODS. HST F775W images and follow-up ACS slitless spectra do not show blue light that would be expected if the supernova were of Type II.

advance information is used to help select one or two likely Type Ia supernovae suitable for follow-up. Each team has three target-of-opportunity triggers and 60 orbits for near-infrared follow-up observations with NICMOS and slitless spectroscopy with ACS. For these searches each supernova lightcurve and its associated search costs roughly 30 HST orbits.

At the time of this conference the SCP had netted a probable $z \sim 1.7$ Type Ia supernova from the first of the four search epochs. The discovery image is show in Figure 2. Since then, several more supernova have been found as part of this program. Unfortunately, all of these supernovae have just the bare minimum of data needed to place them on the Hubble diagram, leaving little extra information for testing for systematic effects in the measurement. A satellite such as $S N A P$ will increase the number of such supernovae by orders of magnitude, but will also have much higher data quality and systematics controls.

\section{Enabling Better Type Ia Standardization}

While Type Ia supernovae have shown tremendous value in revealing the presence of dark energy and exploring its nature, we should be concerned that this purely empirical technique will reach a systematics floor. The single-parameter light-curve width correction method (plus extinction correction) reduces the scatter in Type Ia supernovae at peak to somewhere in the range $0.10-0.15$ mag $[19,12]$. However, there is nothing special about the physical conditions 

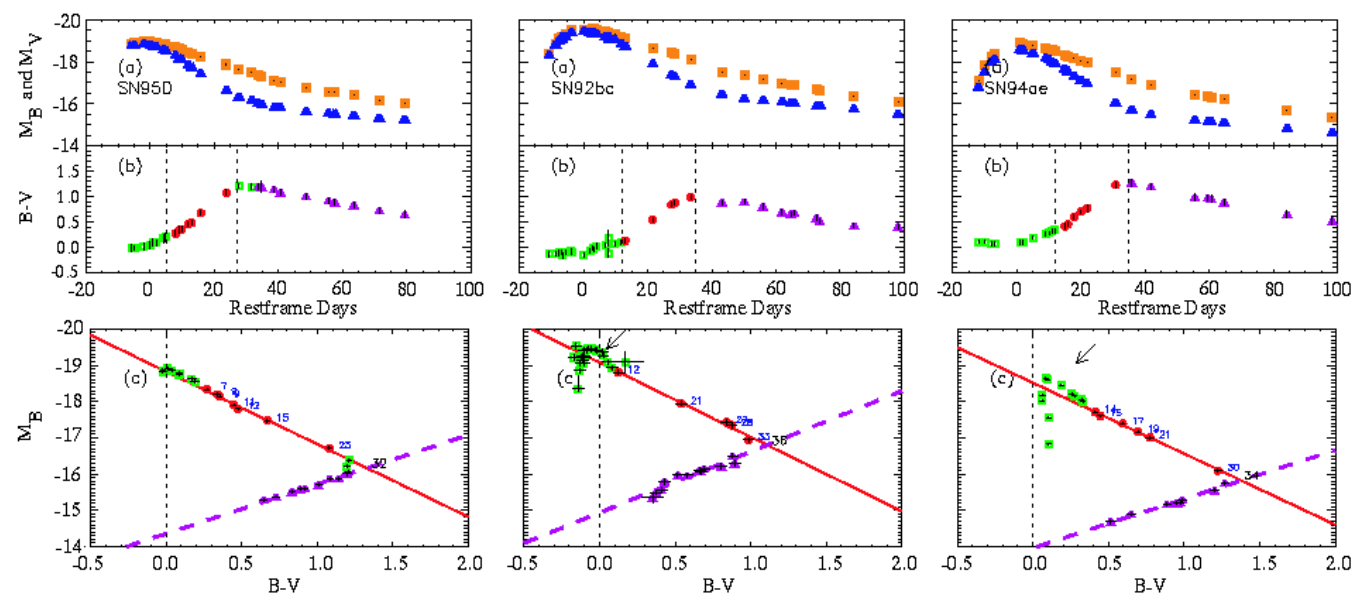

Fig. 3. Illustration of the CMAGIC Type Ia supernova standardization technique for three well-observed Type Ia SNe. The upper row shows the $B$ and $V$ lightcurves, the middle row shows the $B-V$ color curves, and the bottom row shows $B$ versus $B-V$ color. Classical techniques (stretch, $\Delta \mathrm{m}_{15}$, MLCS) use the lightcurves and color curves versus time, whereas CMAGIC uses brightness versus color, which has an amazingly linear region starting about a week after maximum light. (There is also a linear region at late times, during the nebular phase.) The brightness when $B-V=0.6$ is an excellent standard candle [21].

in a supernova when it appears its brightest. Indeed a newly developed technique, CMAGIC, standardizes Type Ia supernovae using the color and magnitude roughly 15 days after maximum, when the supernova reaches a color of $B-V=0.6$ [21]. This method has been shown to reduce the scatter to below 0.10 mag. Thus, it is possible that Type Ia supernovae are more homogeneous at a given color rather than at a given lightcurve epoch. Many more observations of nearby supernovae, focusing on accurate colors with well-determined uncertainties will be a great boon in improving the standardization of Type Ia supernovae. By lowering the remaining scatter, stronger limits are also set on any possible remaining systematics in the Type Ia methodology.

\section{From Science Objectives to Project Design}

The objective of the SNAP mission is to provide the best possible constraint on the time-evolution of the dark energy equation of state. The only demonstrated experimental techniques are astronomical, and of these, the luminosity distance from Type Ia supernovae is by far the most mature. Therefore, it was natural to design a mission focused on supernovae, but as we shall see, the resulting design is sufficiently general to perform exceptionally well for gravitational weak lensing.

Pushing the Type Ia supernova method to its limit requires thousands of su- 
pernovae spanning a wide range in redshift. The present $S N A P$ design targets 2000 normal Type Ia supernovae from redshift 0.1 to 1.7 . It is extremely critical that the photometry be of high quality continuously from low to high redshift, not only to realize the statistical power of the sample but to avoid introduction of systematics errors [4]. Due to the increasing brightness of the terrestrial night sky at redder wavelengths where higher redshift Type Ia supernovae must be observed, coupled with increasing atmospheric water absorption at redder wavelengths, the necessary data quality can be obtained only from space. The broad redshift coverage needed to control systematics further requires detectors at both optical and infrared wavelengths. Telescope optical design and spacecraft size and mass constraints point to a dedicated 2 -m telescope ${ }^{4}$ with a field of view several hundred times larger than that possessed by $H S T$, populated with visible and near-infrared detectors. Further, in order to determine the supernova type, and possible sub-class, a sensitive low-resolution spectrograph is required. Systematics control enabled by spectrophotometric spectra, as well as allowance for spacecraft pointing margin, call for an integral field unit spectrograph.

\section{The Power of Weak Lensing from Space}

Gravitational weak lensing measurements have become increasingly robust over the past decade. Long viewed as a means of constraining $\Omega_{M}$, more detailed theoretical studies - several developed within the SNAP collaboration - indicate that precision weak lensing measurements have the capability to place constraints on the dark energy equation of state similar to those possible with Type Ia supernovae. In particular, weak lensing cross-correlation cosmography - measuring the association between foreground template mass distributions and background weak lensing shear using galaxies located using photometric redshifts - appears to be the most powerful weak lensing approach [2].

Atmospheric turbulence results in poor spatial resolution for high-redshift galaxies observed from the ground. Furthermore, ground-based telescope and camera optics are far from being diffraction-limited. So great care must be taken in removing the effects of seeing, tracking errors, and optical aberrations. Stars provide the reference for removing these effects, but the number of usable stars is limited, due to both the limited size of the isoplanatic patch within which seeing is correlated and difficulty in distinguishing the few stars from the large number of galaxies at faint magnitudes. Errors in these corrections set a limit on the quality that ground-based weak lensing will be able to achieve.

4 For diffraction-limited photometry of faint point sources, exposure time goes as the 4 th power of the telescope aperture 


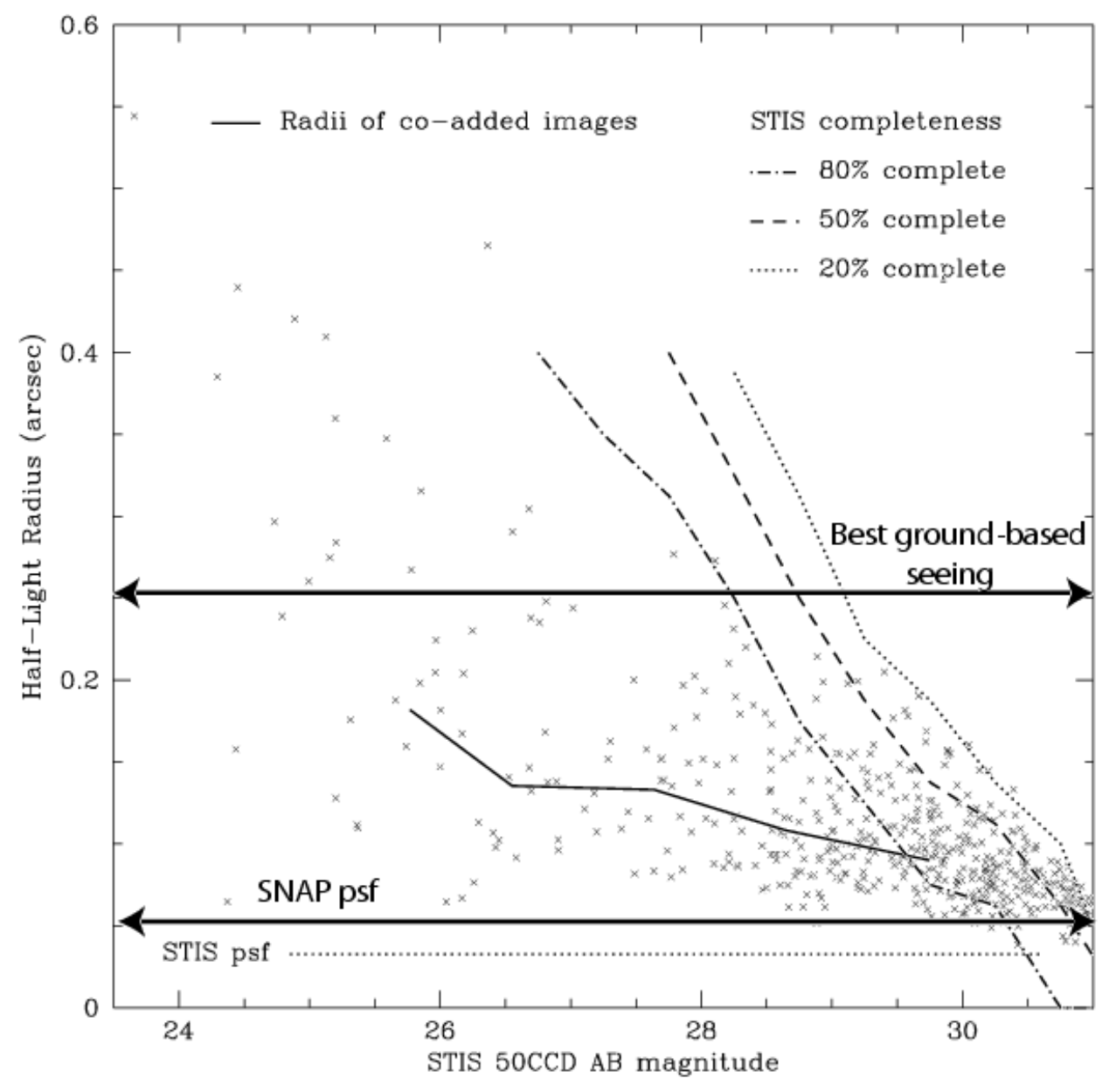

Fig. 4. Measurements of the sizes of galaxies versus magnitude [3], illustrating the large reservoir of faint, distant galaxies which can be spatially resolved from space but not from the ground.

In comparison, space-based wide-field observations resolve many more sources and the remaining corrections for tracking and telescope aberrations are much smaller relative to the sizes of the sources. Figure 4 illustrates the dramatic gain in the number of resolved galaxies in going from ground- to space-based observations. Moreover, in contrast to $H S T, S N A P$ will be placed in a stable thermal environment so that any corrections can be determined using many spatially uncorrelated images. Moreover, the deep optical and infrared photometry from $S N A P$ will provide high-quality photometric redshifts as needed to implement the cross-correlation cosmography method. Therefore, SNAP can provide excellent weak lensing data for constraining changes in the dark energy equation of state [14]. 


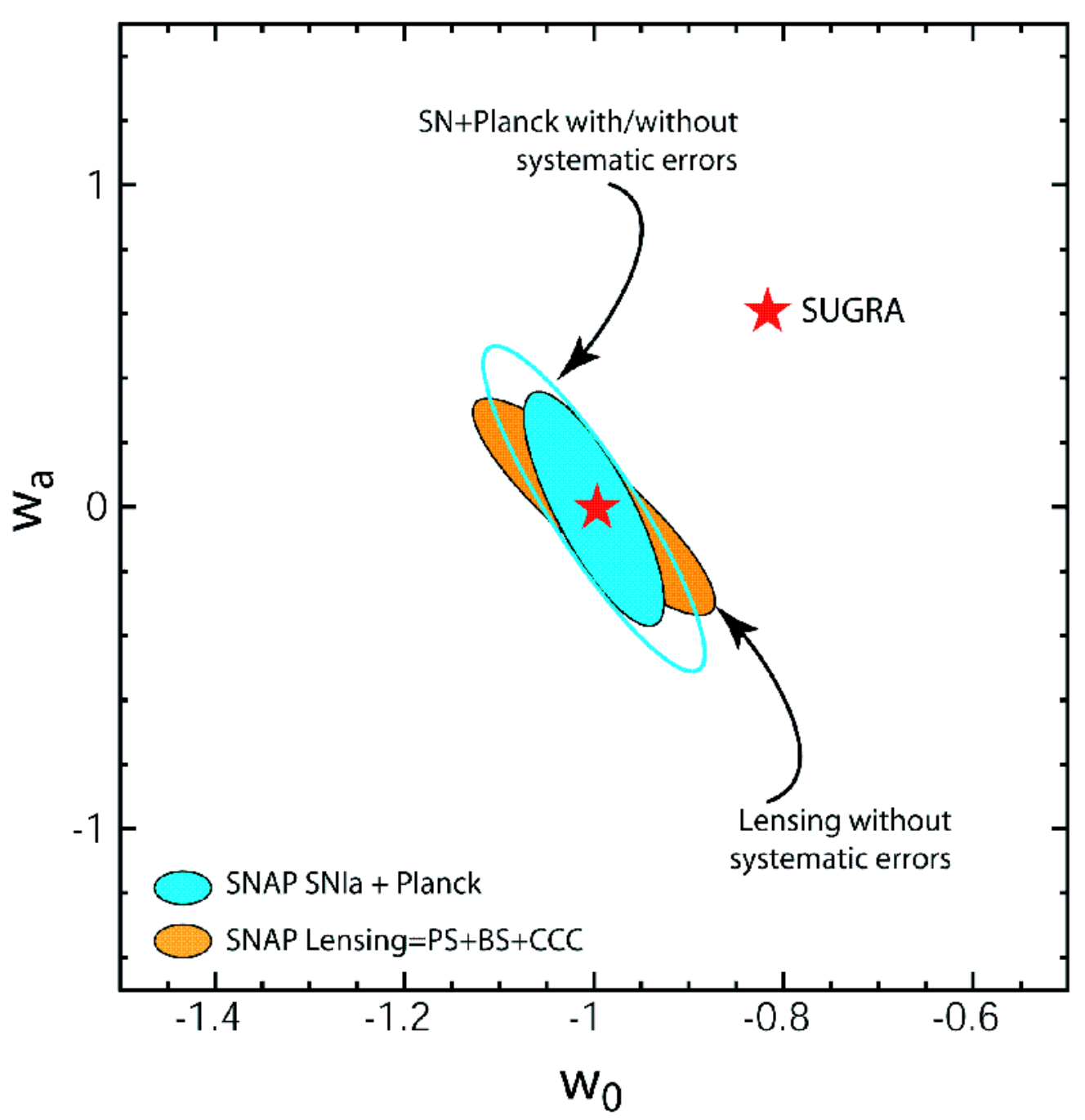

Fig. 5. Individual constraints on the dark energy equation of state and its derivative from the SNAP Type Ia SN program and the weak gravitational lensing program, demonstrating the comparable and complementary power of these two methods when executed with SNAP.

\section{$6 \quad S N A P$ Instrument Concept}

In detail, the SNAP telescope design is a three-mirror anastigmat with a $2-\mathrm{m}$ primary mirror [7]. The optics are silver-coated to maximize the optical and near-infrared throughput and minimize the thermal emissivity. The imager consists of 0.34 sq. degrees paved with red-sensitive CCD's and a scale of 0.10 "/ pixel, and 0.34 sq. degrees paved with $\mathrm{HgCdTe}$ near-infrared detectors with a scale of $0.18 " /$ pixel [6]. The sizes of the detectors are identical and those made of $\mathrm{HgCdTe}$ have a long-wavelength cutoff which reduces the dark current (and sensitivity to thermal emission). These features allow the CCD and $\mathrm{HgCdTe}$ detectors to be mounted in the same focal plane, simplifying 

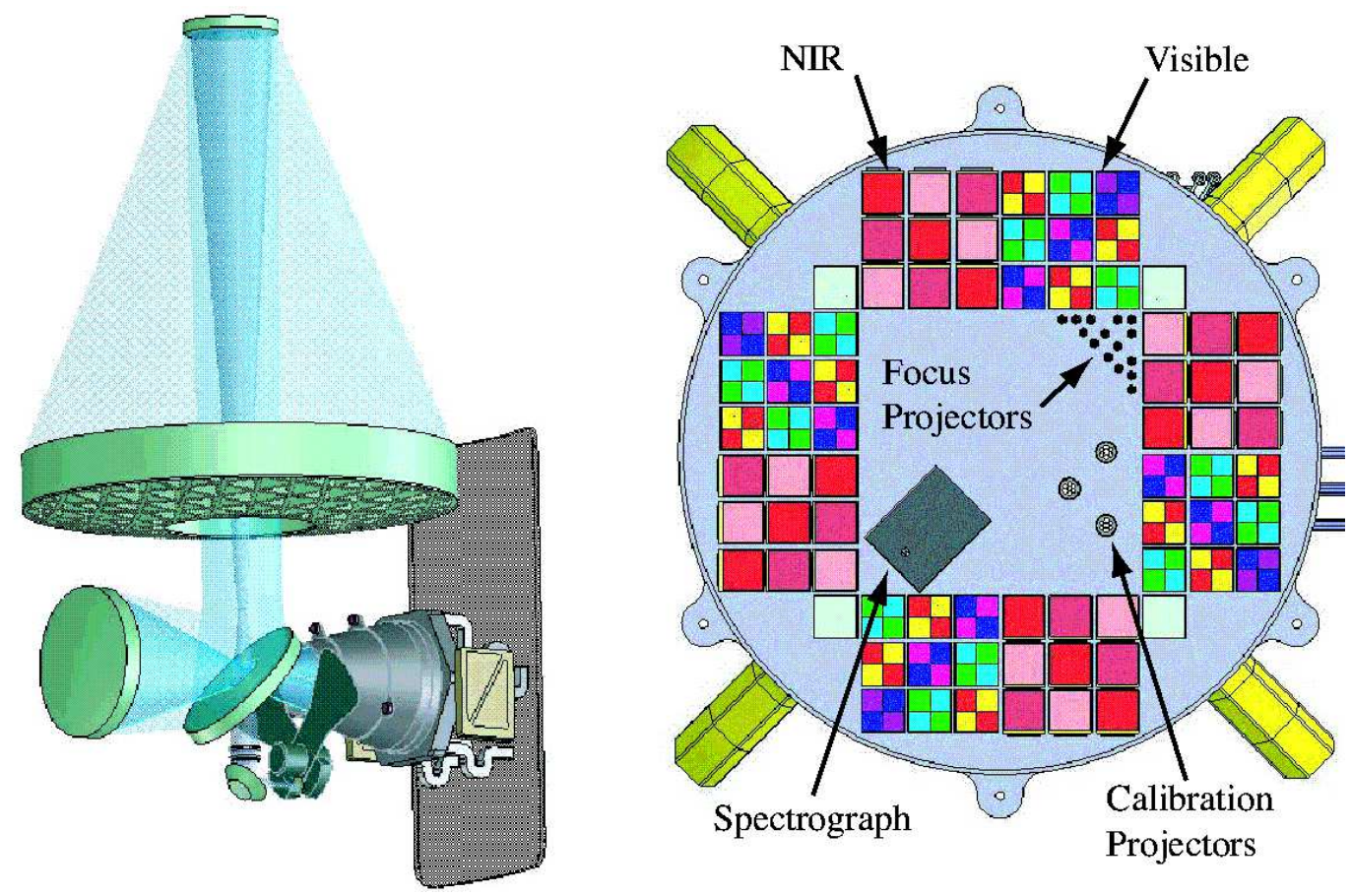

Fig. 6. Left: Layout of the SNAP optical telescope assembly (OTA) [7]. The telescope, shutter, camera (shielded by the cone), electronics, and passive radiator are shown. Right: Layout of the SNAP focal plane [6]. NIR and visible detectors populate a single focal plane. Each detector has its own filter. The filters (and hence detectors) are laid out so that as $S N A P$ passes over a field, each location is observed in all filters. The $S N A P$ spectrograph is mounted behind the imaging focal plane, and is fed by an IFU in the imaging focal plane [13]. Detectors for guiding populate each quadrant, and projectors for focus and calibration are also integrated into the focal plane.

the imager construction, mounting, and alignment. Each detector has its own filter, so a large filter wheel which would otherwise be needed is avoided. Detailed simulations show that photometry of sufficient quality and redshift range is not possible from the ground even under optimistic assumptions.

The $S N A P$ spectrograph has a prism as its dispersing element, providing resolution $R \sim 100$ across optical and near-infrared wavelengths [13]. Higher resolution is unnecessary due to the velocity broadening intrinsic to the spectra of Type Ia supernovae. Again, the optical detector is a red-sensitive CCD while the near-infrared detector is $\mathrm{HgCdTe}$ with a long-wavelength cut-off near $1.7 \mu \mathrm{m}$. The spectrograph is fed by an image slicer integral field unit. The field is $6^{\prime \prime} \times 3^{\prime \prime}$, wth the latter dimension being divided into 40 slices. Detailed simulations show quite dramatically that spectroscopy of comparable quality is not possible from the ground, even with a thirty meter telescope with adaptive optics. 
Table 1

Nominal parameters of the SNAP surveys

\begin{tabular}{|l|c|c|c|c|}
\hline Survey Mode & Areal Coverage & Depth & $\begin{array}{c}\text { Resolved Galaxy } \\
\text { Surface Density } \\
\left(\# \text { arcmin }^{-2}\right)\end{array}$ & $\begin{array}{c}\text { Number of } \\
\text { Resolved Galaxies }\end{array}$ \\
\hline Deep SNe & 15 & 30.3 & 250 & $10^{7.0}$ \\
Wide & $300-1000$ & 27.7 & 100 & $10^{8.5}$ \\
Panoramic & $7000-10000$ & 26.7 & $40-50$ & $10^{9.0}$ \\
\hline
\end{tabular}

\section{Conclusion}

The nature of dark energy is a fundamental question, which astronomical observations can address. Type Ia supernova and weak lensing are the most powerful techniques yet developed for studying dark energy. Reaching the requisite statistical and systematics accuracy with these techniques requires a widefield imager in space. SNAP represents a very advanced concept for such a widefield imager, having an instrument suite which is both powerful and versatile. Table 1 details this power for the Type I supernova survey, a nominal wide-field weak lensing survey, and a possible panoramic weak lensing survey. The planned dark energy program for the Joint Dark Energy Mission (JDEM) will produce a treasure trove of data for archival study. Guest Observer programs that could further exploit such a powerful instrument are envisioned in the JDEM concept. The following conference presentations describe many of the scientific lines of inquiry which would be greatly advanced with a facility such as SNAP.

\section{References}

[1] Aldering, G. et al. 2004, astro-ph/0405232

[2] Bernstein, G. \& Jain, B. 2004, ApJ, 600, 17

[3] Gardner, J. P. \& Satyapal, S. 2000, AJ, 119, 2589

[4] Kim, A. G., Linder, E. V., Miquel, R., \& Mostek, N. 2004, MNRAS, 347, 909

[5] Knop, R. A. et al. 2003, ApJ, 598, 102

[6] Lampton, M. L. et al. 2002a, Proc. SPIE, 4854, 632

[7] Lampton, M. L. et al. 2002b, Proc. SPIE, 4849, 215

[8] Percival, W. J. et al. 2002, MNRAS, 337, 1068

[9] Perlmutter, S. et al. 1997, ApJ, 483

[10] Perlmutter, S. et al. 1998, Nature, 391

[11] Perlmutter, S. et al. 1999, ApJ, 517, 565

[12] Phillips, M. M. et al. 1999, AJ, 118, 1766 
[13] Prieto, E. et al. 2002, Proc. SPIE, 4850

[14] Rhodes, J. et al. 2004, Astroparticle Physics, 20, 377

[15] Riess, A. G. et al. 1998, AJ, 116, 1009

[16] Riess, A. G. et al. 2004, ApJ, 600, L163

[17] Spergel, D. N. et al. 2003, ApJS, 148, 175

[18] Tonry, J. L. et al. 2003, ApJ, 594, 1

[19] Tripp, R. 1998, A\&A, 331, 815

[20] Sullivan et al. 2003, MNRAS, 340, 1057

[21] Wang, L., Goldhaber, G., Aldering, G., \& Perlmutter, S. 2003, ApJ, 590, 944 